Revista de Matemática: Teoría y Aplichciones 2000 7(1-2) : 229-244

CIMPA - UCR ISSN: 1409-2433

\title{
EL ANÁLISIS MULTIVARIADO DE DATOS COMO HERRAMIENTA EN LA EXPLORACIÓN DE MODELOS EN LA COMPRENSIÓN DE VARIABLES PSICOLÓGICAS*
}

\author{
Carlos R. Seijas ${ }^{\dagger}$
}

Recibido: 1 Setiembre 2000

\begin{abstract}
Resumen
La psicología como ciencia de la conducta en un estado preparadigmático necesita del apoyo de modelos teóricos que validen y confiabilizen los constructos que esta rama del conocimiento utiliza para explicar los fenómenos que le competen estudiar. Para sustentar los avances en la construcción de modelos provenientes de diversos campos de la psicología, se presenta la aplicación de herramientas del análisis multivariado de datos tales como el Análisis de Componentes Principales (ACP), el Análisis Factorial de Correspondencias (AFC) y el Análisis Factorial Discriminante (AFD), en el estudio de variables como la predicción del desempeño universitario de acuerdo a variables clásicas de la psicometría (Seijas, 1998a) y a variables cognoscitivas (Seijas, 1999b), los estilos de aprendizaje (Seijas, 1998b), la autoestima (Seijas, 1999a), y finalmente variables transculturales como son el individualismo y el colectivismo (Seijas, 1999c). Con esto se persigue mostrar la utilidad que el análisis multivariado de datos puede prestar a las ciencias de la conducta en su meta de forjar constructos teóricos con poder explicativo de la realidad, para que puedan ser de instrumentales operativos en el quehacer diario de todos los interesados en el análisis y manejo de la conducta.
\end{abstract}

Palabras clave: Análisis Multivariado de Datos, Modelos Teóricos, Psicología, Métodos Matemáticos, Aplicaciones Matemáticas.

\footnotetext{
* Se agradece el apoyo y colaboración del Director de la Escuela Superior de Psicología de la Universidad Francisco Marroquín Dr. Luis A. Recinos, así como de los alumnos que ayudaron en la recolección y calificación de los instrumentos utilizados. Igualmente se agradece a la Escuela de Matemáticas de la Universidad de Costa Rica y a los miembros del Programa de Investigación en Modelos y Análisis de Datos por permitir la utilización del paquete de Análisis de Datos PIMAD 3.0 en especial al Dr. Javier Trejos y al Dr. Oldemar Rodríguez Rojas.

${ }^{\dagger}$ Universidad Francisco Marroquín, Guatemala, Guatemala. Dirección personal: 29 Ave. 28-77 Zona 5, Colonia Santa Ana, Ciudad de Guatemala, Guatemala. E-Mail: cseijas@ufm.edu.gt
} 


\begin{abstract}
Psychology, as a behavioral science in a preparadigmathic state, needs the support of theoretical models that validate the constructs that it uses for explaining the phenomena it studies. For sustaining the advances in the construction of models that arise in different domains of the Psychology, we present the application of multivarate data analysis tools such as Principal Component Analysis, Correspondence Analysis and Discriminant Analysis. We study variables such as prediction of university performance according to classical variables in Psychometrics (Seijas 1998a) and sognoscitive variables (seijas 1999b), learning style (Seijas 1998b), autoesteem (Seijas 1999a), and finally transcultural variables such as individualism and collectivism (Seijas 1999c). We want to show the usefulness that multivariate data analysis can give to behavioral sciences for making theoretical constructs with power to explain the reality, in such a way that the can be operational tools in the people interested in analyzing and handling the behavior.
\end{abstract}

Keywords: Multivariate Data Analysis, Theoretical Models, Psychology, Mathematical Models, Mathematical Applications.

Mathematics Subject Classification: 62P15

\title{
1 Introducción
}

Las ciencias empíricas se basan en la investigación de datos que pueden validar hipótesis. Popper (1934) decía que puede llamarse científica a la disciplina que cuenta con medios para refutar una proposición. Popper insiste en que basta una observación para descartar una ley. Como no se puede elaborar una lista de todas las observaciones a las que se aplica una ley, es imposible afirmar que sea verdadera. El descubrimiento de hechos que contradigan una teoría implica la elaboración de una nueva teoría. Cada nueva teoría permite una nueva conceptualización de problemas que pueden llegar a modificar por completo la problemática, dado que la ciencia procede por aproximaciones sucesivas. La actividad del investigador tiene como eje la teoría y, de manera más general, el sistema explicativo, aunque con frecuencia sucede, sobre todo en los terrenos poco explorados, que la investigación se inicia a través de observaciones o interrogantes: una pregunta ocupa el lugar de la predicción. Entonces el propósito de la experiencia es recopilar datos que permitan contestar la pregunta. La actividad del investigador no se modifica en lo fundamental. Sea cual fuere el marco en que se sitúe, la investigación tiene un propósito descriptivo o explicativo.

La evolución de las ciencias en general, estádando como resultado mayor rigor en la aplicación de métodos cada vez más útiles y cercanos a la exactitud. Sin ellos no serían posibles la evaluación y la comparación de resultados, la comprobación de hipótesis y, por último, la postulación de teorías y modelos. Técnicas y métodos constituyen la piedra angular y, en parte, el mecanismo de consenso, de aceptación de cualquier empresa científica. Las ciencias de la conducta, y en especial la psicología, que aún es considerada por algunos autores como ciencia preparadigmática en el modelo planteado por Kuhn (Leahey, 1982), necesitan en gran medida el apoyo de modelos que le permitan tener un acercamiento más profundo a los constructos teóricos de los cuales constantemente se 
plantea su validez y confiabilidad, para que encuentren eco en el quehacer diario de los profesionales de las ciencias de la conducta, entre ellos los psicólogos,, en sus diversas ramas de intervención.

Los modelos teóricos han permitido el avance de las ciencias de la conducta en la medida en que éstos modelos van respondiendo cada vez más y de mejor forma a la realidad. Para conseguir este apego a la realidad, es necesario contrastar los modelos que han surgido de la observación de uno o varios estudiosos de la conducta, y realizar trabajo experimental a través de medios como son las investigaciones de campo, tomando un instrumento, que parte del supuesto de una validez de contenido apropiada, que serviráa la vez para dar el primer paso en la comprobación del modelo teórico con la realidad.

Con el fin de mostrar la importancia del uso del análisis multivariado de datos en la comprensión y comprobación de algunos modelos de la psicología, se presentan los resultados de algunas investigaciones sobre las temáticas que cubren el desempeño universitario de acuerdo a variables clásicas de la psicometría (Seijas, 1998a) y a variables cognoscitivas (Seijas, 1999), los estilos de aprendizaje(Seijas, 1998c), la autoestima (Seijas, 1999a), y finalmente variables transculturales como son el individualismo y el colectivismo (Seijas, 1999b).

\section{Modelo 1: el desempeño universitario desde la óptica de las variables psicométricas clásicas}

En este estudio se investigósobre la importancia de variables psicométricas clásicas en la predicción del desempeño de los estudiantes universitarios, tales como Habilidad Verbal, Habilidad Matemática, y el Coeficiente Intelectual.

\section{Método}

\section{Participantes}

El grupo de estudio fue formado por los estudiantes del departamento de psicología de la Universidad Francisco Marroquín, en el período de 1994 a $1998(N=337)$ de los cuales el $88 \%$ son de Sexo Femenino $(n=297)$ y el $12 \%$, Masculino $(n=40)$

\section{Instrumentos}

Los instrumentos utilizados en la obtención de datos fueron los tests de aptitudes de Habilidad Verbal y Habilidad Matemática en sus formas H y J creados por el personal del Instituto de Investigaciones Educativas de la Universidad del Valle de Guatemala (1970), asícomo la prueba de Inteligencia Otis Superior forma A (Otis, 1987).

\section{Modelos de análisis}

Análisis de Componentes Principales (ACP), Análisis Factorial de Correspondencias (AFC). 


\section{Procedimiento}

La aplicación de las pruebas, estuvo a cargo del Departamento de Investigaciones Educativas de la Universidad del Valle de Guatemala (UVG).

En cuanto a la recopilación de información, ésta se obtuvo de los archivos de estudiantes del departamento de psicología. Los datos se vaciaron a mano en hojas diseñadas de acuerdo al Grupo en que se clasificaron a los individuos. Se obtuvieron las variables de los punteos en Habilidad Verbal (HV), Habilidad Matemática (HM), en sus formas brutas (HVPB y HMPB) como rango percentiles (HVRP; HMRP). Derivado de los punteos en HV y HM se obtienen los punteos en Aptitud Académica tanto en forma bruta (AAPB) como en rango percentil (AARP). Los punteos obtenidos por los individuos en cada una de las fechas establecidas son entregados a cada departamento de la UFM por el Centro de Investigaciones Educativas de la UVG. El único punteo que se calculófue el de AAPB (Aptitud Académica Punteo Bruto), dado por la suma aritmética de los punteos brutos obtenidos en HVPB (Habilidad Verbal Punteo Bruto) y HMPB (Habilidad Matemática Punteo Bruto). La variable Sexo también fue obtenida a través de los nombres de los individuos participantes. Este grupo cuenta además con la variable experimental Otis, que corresponde al coeficiente intelectual obtenido en la prueba Otis Superior Forma A. Se agregóel Promedio obtenido por los estudiantes en el primer semestre de su Carrera.

\section{Resultados y discusión}

En la Figura 1 se ve el Círculo de Correlaciones del ACP, en este se observa como Otis se encuentra cercano a Promedio, al igual que la Habilidad Verbal tanto en bruto como en percentiles. La Aptitud Académica (AAPB y AARP) se encuentra sobrepuesta a la primera componente. En la Figura 2 se presenta el Plano Principal del AFC, en el cual se puede apreciar que los estudiantes (representados por el símbolo $\star$ ) se dispersan junto con su Promedio y el Otis hacia el Cuadrante II (derecha de la gráfica), y Habilidad Matemática y verbal aparecen invertidos sobre el Eje 2, mientras la Aptitud Académica permanece sobre el Eje 1.

Las pruebas de Habilidad Verbal y numérica forma H, presentaron características particulares. De acuerdo a los resultados del ACP (Análisis de Componentes Principales), hay que resaltar un paralelismo entre la primera componente principal y la variable AA tanto en punteo bruto como percentil, mientras que la segunda componente principal guarda una relación continua, que va de la variable HV a la de HM. Sobre la base de estas relaciones, se esperaría que la primera componente principal represente los punteos de aspirantes a alumnos como de los alumnos ya inscritos, como se puede observar a través del ACP de los individuos en los tres grupos. Es de resaltar el hecho que la variable Promedio, se encuentra cercana al eje de la segunda componente principal al que se puede denominar "continuo verbal-matemático", con una tendencia hacia Habilidad Verbal y dentro del marco que incluye a AA a la que se comprenderácomo el "continuo éxito-fracaso" de acuerdo a lo que éste índice representa en la literatura clásica de las pruebas psicométricas de aptitudes (Anastasi y Urbina, 1998).

En conjunto con los resultados de las matrices de correlación en los Grupos Dos y Tres, 


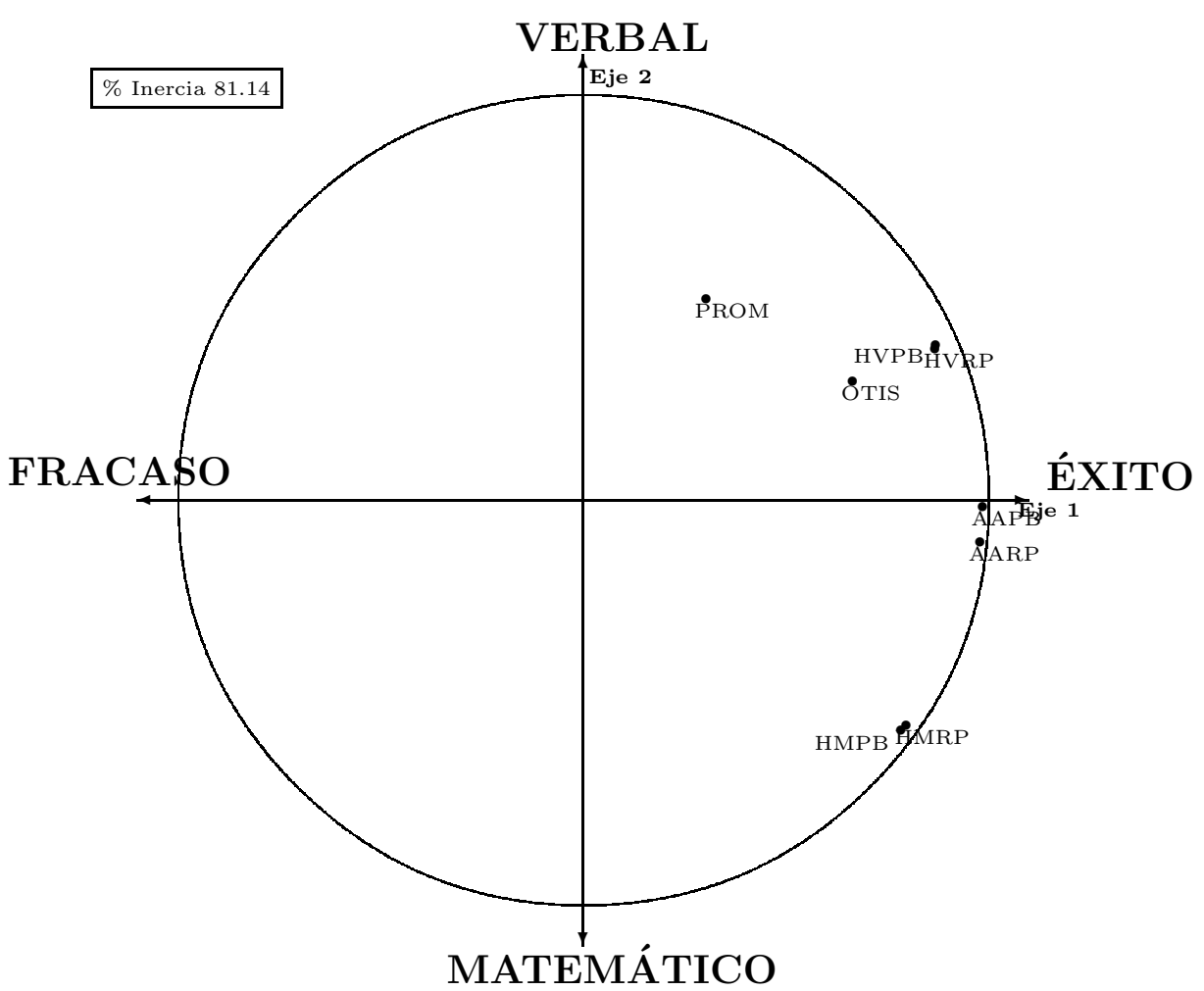

Figura 1: Modelo de Rendimiento Académico en base al Círculo de Correlaciones del ACP.

se ve que las Pruebas Forma $\mathrm{H}$ verbal y matemático, demuestran coherencia interna, lo que indica validez de contenido del instrumento (Solomon de Dougherty, 1989), aunque HM resultóbaja en comparación con los demás, lo que indica que AA conlleva más peso del área verbal que numérica. La prueba Otis presentórelación con AA y HV lo que presenta un peso mayor en dicha prueba en el área verbal. Con respecto a la variable dependiente Promedio, si bien todos los coeficientes de correlación fueron significativos, el más alto lo presentóHV en el Grupo Tres y en el Dos con $\mathrm{r}=.31$ y .28 respectivamente. Esto indica que las variables independientes síse relacionan con la dependiente.

\section{Modelo 2: Análisis del Desempeño Académico a través de Variables Cognoscitivas}

Con el fin de darle seguimiento a las propuestas recomendadas en la anterior investigación, se realizóel presente estudio; que persiguióobservar si existen variables cognoscitivas relacionadas con el éxito académico. 


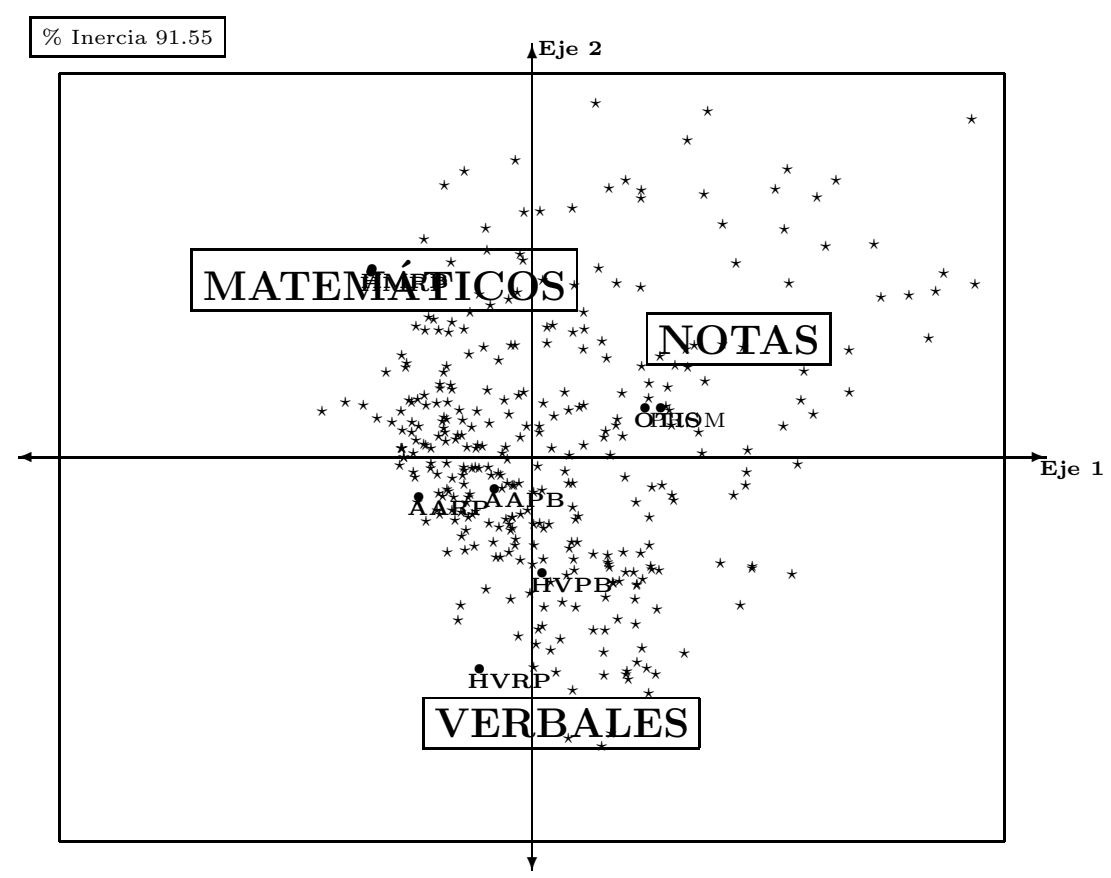

Figura 2: Grupos de Habilidades obtenido del Plano Principal del AFC.

\section{Método}

\section{Participantes}

Se obtuvo una muestra de 64 estudiantes de todos los años de la Licenciatura en Psicología del Departamento de Psicología de la Universidad Francisco Marroquín.

\section{Instrumentos}

Para poder evaluar las variables predictoras se escogieron las pruebas siguientes: Otis Superior Forma A, Perfil de Estilos de Aprendizaje de Kolb (1977), Lateralidad Cerebral de Alder (1995) , Coeficiente Emocional de Bar-On (1996), LASSI (Inventario de Estrategias de Estudio y Aprendizaje) de Winstein, Palmer y Schulte (1987)

\section{Modelos de Análisis}

Análisis de Componentes Principales (ACP), Análisis Factorial de Correspondencias (AFC). 


\section{Procedimiento}

Las variables analizadas fueron Primer Promedio (PP) y Promedio Acumulado (PA), Coeficiente Intelectual (CI). De la prueba de Perfil de Estilos de Aprendizaje: Experiencia Concreta (EC), Observación Reflexiva (OR), Conceptualización Abstracta (CA) y Experimentación Activa (EA). Coeficiente Emocional (CE). De la prueba de lateralidad cerebral se evaluaron las variables del los hemisferios Izquierdo (IZQ y Derecho (DER). De la prueba de Estrategias de Estudio y Aprendizaje (LASSI): Actitudes (ATT); Motivación (MOT); Utilización del tiempo (TMT; Ansiedad (ANX); Concentración (CON); Procesamiento de la información (INP); Selección de ideas principales (SMI); Uso de ayudas especiales (STA); Auto Evaluación (SFT); Estrategias para tomar exámenes (TST).

\section{Resultados y discusión}

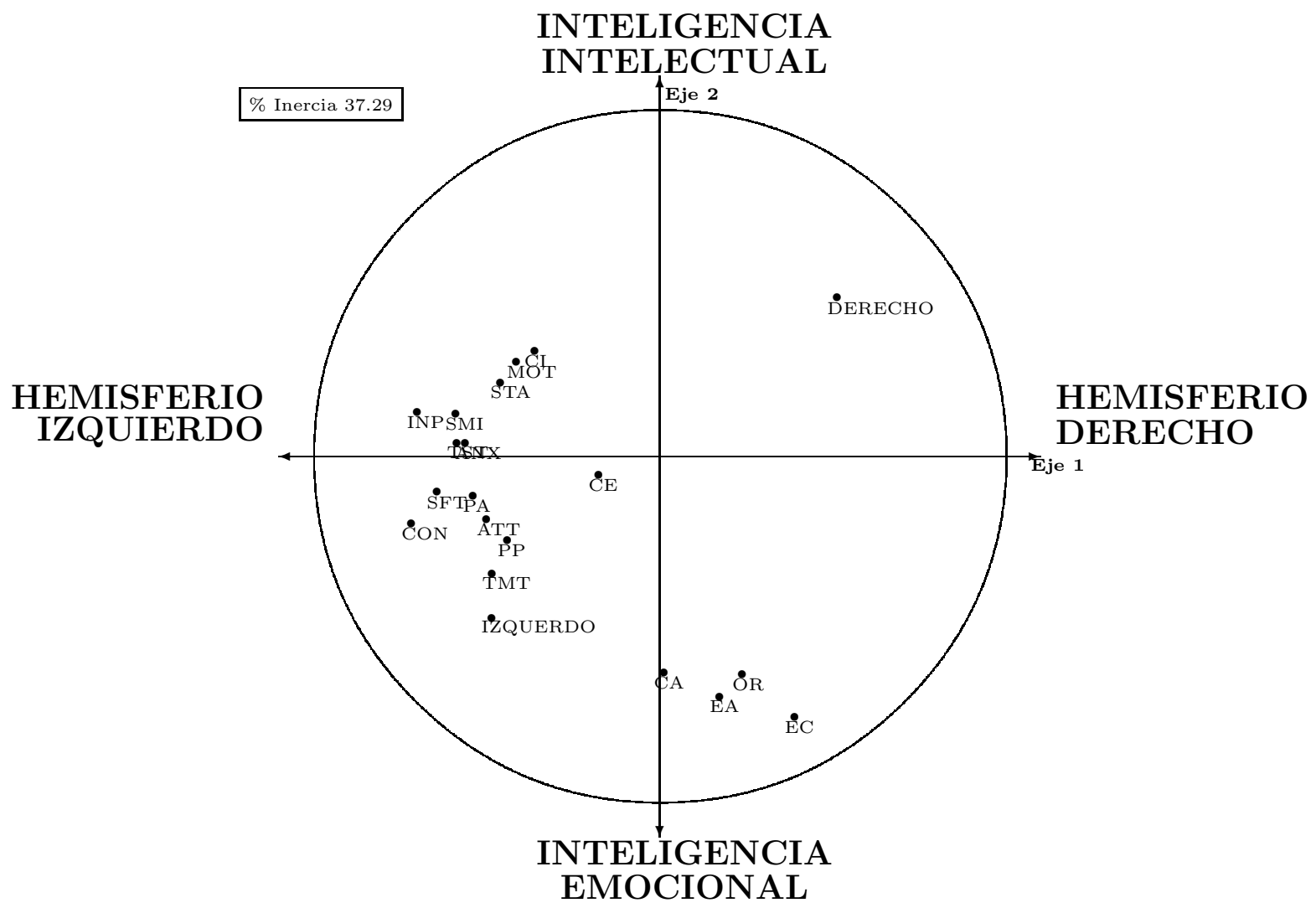

Figura 3: Modelo obtenido del círculo de correlaciones del ACP para las variables cognoscitivas, Coeficiente Intelectual, Primer Promedio y Promedio Acumulado.

Bajo la óptica de Luthans, Hodgetts y Rosenkrantz (1988), las personas exitosas pasan más tiempo formando redes sociales, que haciendo sus tareas, esto último le corresponde a las personas eficientes. Según el modelo planteado por estos autores, la forma tradicional del aprendizaje corresponde a las variables: Utilización de Tiempo (TMT), Uso de ayudas especiales (STA) y Estrategias para Tomar exámenes (TST); la comunicación 
a: Coeficiente Intelectual (CI), Procesamiento de Información (INP) y Selección de Ideas Principales (SMI); las relaciones interpersonales se refieren a las variables: Motivación (MOT), Actitudes (ATT), Ansiedad (ANX) y Auto Evaluación (SFT). La creación de redes al Coeficiente Emocional (CE)

De acuerdo a la comparación anterior, el estudiante con éxito es aquel que presenta un alto CE (Coeficiente Emocional), por lo que su área de desempeño se encuentra en la formación de redes sociales, lo que implica que obtendráresultados deficientes en su rendimiento académico. El estudiante eficaz es el que obtiene mejores resultados, por lo que sus cualidades son un alto CI y un adecuado uso de su tiempo; y pude ser que debido a su dedicación académica, no tenga redes sociales extensas. De ahí que preguntemos qué se prefiere: un estudiante exitoso o uno eficiente.

\section{Modelo 3: estilos de aprendizaje y lateralidad cerebral}

\section{Método}

\section{Participantes}

Se utilizó una muestra de 18 participantes seleccionados del tercer año del Departamento de Psicología de la Universidad Francisco Marroquín.

\section{Instrumento}

Se utilizó la prueba denominada Perfil de Estilos de Aprendizaje (Kolb, Rubin y McIntyre, 1977), integrada por un inventario para evaluar el método de aprendizaje y una lista de los diferentes conceptos con sus sinónimos.

La prueba de lateralidad cerebral utilizada fue desarrollada por Alder (1995)

\section{Procedimiento}

Las variables independientes utilizadas fueron: Modos de Aprendizaje, Estilo de Aprendizaje. Las Variables Dependientes: Lateralidad Cerebral.

\section{Modelos de Análisis}

Análisis de Componentes Principales (ACP), Análisis Factorial de Correspondencias (AFC), Análisis Factorial Discriminante (AFD).

\section{Resultados y discusión}

Antes de entrar en cualquier análisis, debe advertirse que las conclusiones aquíexpresadas, son en todo caso tentativas, y en ningún momento prescriptivas. Puesto que, con una muestra tan pequeña, cualquier conclusión es aventurada.

Los datos demuestran que la relación en la prueba de lateralidad una correlación negativa perfecta entre Izquierdo y Derecho $(r=-1)$. Aunque es difícil encontrar una relación negativa perfecta entre dos variables cualesquiera que sean, en este caso se comprende 
dicho resultado, viendo que al conceptualizar la lateralidad cerebral en dos, Izquierdo es lo Contrario de Derecho, por lo que puede indicar que la prueba permite un cierto nivel de validez convergente.

En cuanto a la relación entre los cuatro modos de aprendizaje Experiencia concreta (EC), Observación Reflexiva (OR), Conceptualización Abstracta (CA) y Experimentación Activa (EA), que se daba una relación fuerte entre CA y OR que crean el cuadrante IV del plano propuesto por (Kolb, Rubin y McInyre, 1977). La contraparte de OR según Kolb, Rubin y McInyre es EC lo que se mostrógráficamente en el plano del correlacione del ACP. Los datos entre CA y EC permiten ver una relación negativa casi perfecta como en el caso de CA y OR. En cuanto al continuo quedóal centro entre Izquierdo y Derecho guardando por tanto una relación casi nula entre estas variables. El AFD presenta una clasificación de cuatro grupos que siguiendo de la mano de la teoría de Kolb, Rubin y McInyre que pueden pensarse en los Convergentes, Divergentes, Asimilativos y Adaptativos. Los cuadrantes pueden clasificarse de la siguiente forma Cuadrante I Convergente, Cuadrante II Asimilativo, Cuadrante III Divergente, Cuadrante IV Adaptativo. El continuo Derecha-Izquierda delimita los grupo de la siguiente forma Derecha Asimilativo y Divergnetes, Izquierdo Convergentes y Adaptativos.Los datos encontrados permiten darle una aprobación experimental a la teoría propuesta por Kolb, Rubin y McInyre (1977). Encontrando que en la muestra de participantes de psicología los cuatro grupos de estilos de aprendizaje se dieron. La propuesta nueva es la de revisar si el plano propuesto por los teóricos coincide con la práctica, y en realidad parece que no. Esto se evidencia gracias al Análisis Multivariado de Datos, cuyos resultados permitieron reclasificar a los individuos en grupos de acuerdo a sus resultados.

En lo que respecta a la variación de la lateralidad cerebral en todas las pruebas se encontró una relación inversamente proporcional entre un plano y el otro. Lo que plantea la existencia de un plano continuo entre izquierdo y derecho, enfatizado por la segunda prueba que permite evaluar la lateralidad en un continuo y no de forma segmentada. Al introducirla entre las modalidades del aprendizaje se corroboró que la lateralidad cerebral derecha acompaña a la modalidad OR y EC, mientras que la lateralidad izquierda acompaña a los modos CA y EA. En relación con los estilos de aprendizaje se verificó nuevamente que como se propuso los estilos Divergentes y Convergentes así como los Adaptativos y Asimilativos se vieron segmentados por un tercer axis que fue la lateralidad cerebral, Derecha para los Divergentes y Asimiladores que tienden a ser más creativos y sociales, mientras que el hemisferio dominante para los convergentes y adaptaivos fue el izquierdo, puesto que sus modos de aprendizaje les exigen más conceptualización abstracta asícomo una experimentación activa. Lo que nos podría llevar a pensar en utilizar esta herramienta para seleccionar alumnos para la universidad o trabajadores con habilidades especificas. 


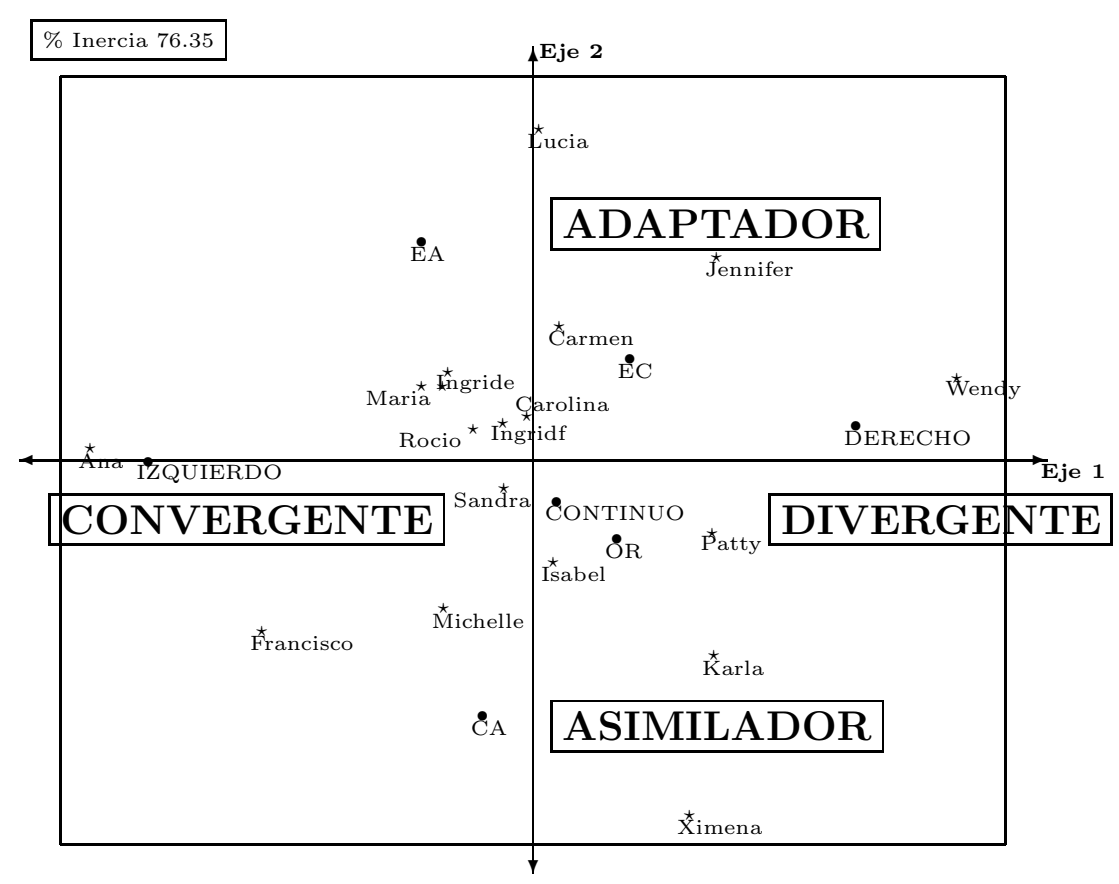

Figura 4: Modelo de Estilos de Aprendizaje derivado del AFC.

\section{Modelo 4: autoestima en estudiantes de economía}

\section{Método}

\section{Participantes}

El grupo de participantes estuvo compuesto por 254 estudiantes de la Facultad de Economía de la Universidad Francisco Marroquín. De estos 139 correspondían al sexo Femenino (55\%) y 115 al sexo Masculino (45\%). La edad de los participantes estaba comprendida entre los 16 y 32 años, con una Media de $19.8(D E=1.78)$. La distribución por semestres se encontróde la siguiente forma, para Primer semestre 96 alumnos (37.8\%); Segundo, 56 (22.05\%); Tercero $66(25.98 \%)$ y Cuarto $36(14.17 \%)$.

\section{Instrumento}

En esta investigación se definióla autoestima como el sentido de autovaloración multidimensional que una persona experimenta en las diferentes áreas de su vida. Para evaluar la autoestima de los sujetos, se utilizóel Five Scale Test of Self-Esteem for Children elaborado por Pope, McHale y Craighead (1988). El cuestionario adaptado evalúa seis áreas de autoestima total, que se obtiene sumando las puntuaciones obtenidas en cada una de 


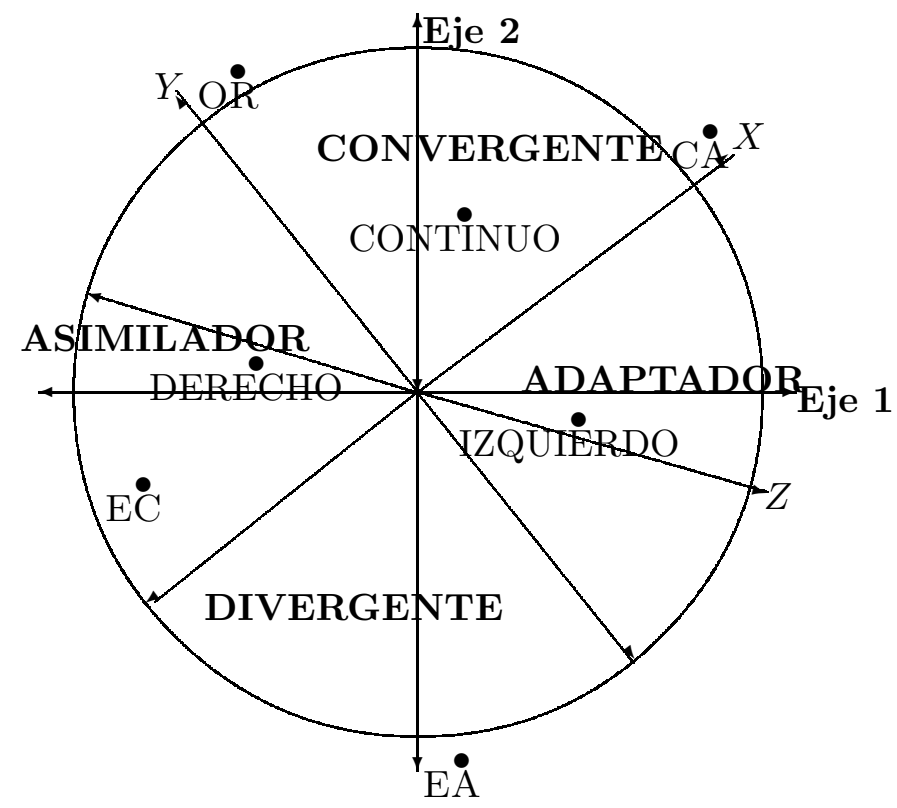

Figura 5: Modelo tridimensional de los estilos de aprendizaje y lateralidad cerebral obtenido del Círculo de correlaciones del AFD.

las áreas específicas. Este cuestionario consta de 60 ítems que constituyen afirmaciones relacionadas con autoestima, para las cuales el sujeto debe responder en quégrado piensa o se siente de esa manera. Los ítems de este test se miden en escala de intervalo de tipo Lickert, puntuando 2 puntos si el sujeto contesta que "casi siempre" se siente de esa manera, 1 punto si "a veces" se siente de esa manera, y 0 puntos si "casi nunca" se siente de esa manera. La sumatoria de las seis áreas constituye la autoestima total de cada sujeto. El punteo es directamente proporcional al grado de autoestima, de tal manera que a mayor puntaje, mayor nivel de autoestima.

\section{Modelos de análisis}

Análisis de Componentes Principales (ACP).

\section{Procedimiento}

Todos los cuestionarios fueron aplicados en los respectivos horarios de clase por los maestros que impartían el curso en el que se encontraban los alumnos de la facultad de economía, según su programación. Posteriormente los catedráticos devolvieron los cuestionarios ya contestados a la catedrática interesada en investigación, quien posteriormente los entregóal profesional encargado del análisis e interpretación de los resultados.

\section{Resultados y discusión}

Al perfilar los resultados encontrados en las correlaciones Producto Momento de Pearson, se puede explorar un modelo que permite explicar en gran parte las relaciones que guardan 


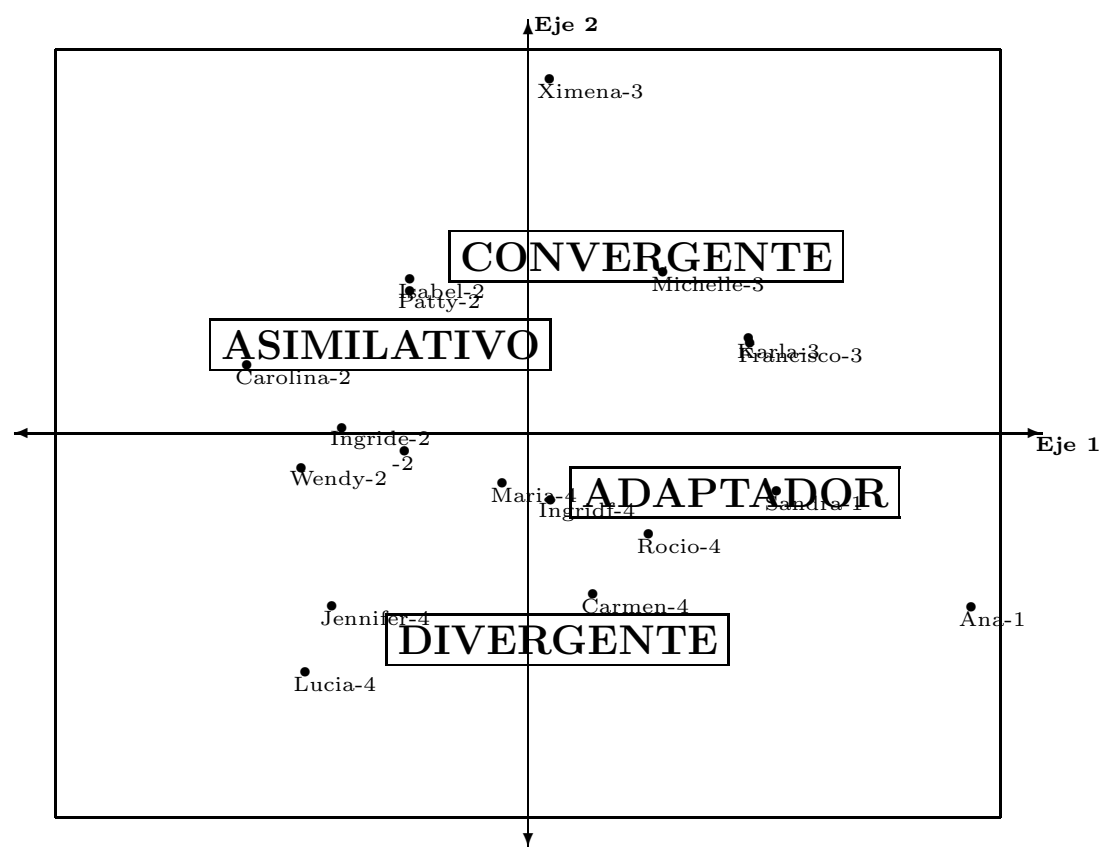

Figura 6: Modelo de grupos de aprendizaje sobre el plano principal del AFD.

las subescalas. La Figura 1 presenta el círculo de correlaciones del Análisis de Componentes Principales, del cual se observa el continuo de dos vertientes, las variables que se pueden denominar relacionadas con la autoestima debida a factores externos (parte superior), y las variables relacionadas con factores internos, propios a la persona (parte inferior). Con este modelo se puede categorízar a la autoestima como construida por factores propios del ambiente y de la persona, es decir, la autoestima es función del Ambiente y de la Persona $\left(A_{e}=f\left(A_{m} \cdot O_{r}\right)\right)$.

De esta forma, desde la Perspectiva de la Teoría del locus de Control de Rotter (1966), se encuentra en este modelo, relacionados a la Autoestima Externa, los factores de Autoestima Social, Global, e Imagen Corporal; mientras que a la Autoestima Interna se vinculan las subescalas de Autoestima Académica, Familiar y Moral. Esta clasificación evidencia las áreas que el estudiante puede percibir como bajo su control (Interna), y fuera de su control (Externa). Puesto que el locus de referencia varia, de acuerdo a la percepción que tenga la persona de que dichos factores están bajo su influencia, o bien dependen en mayor medida del medio. 


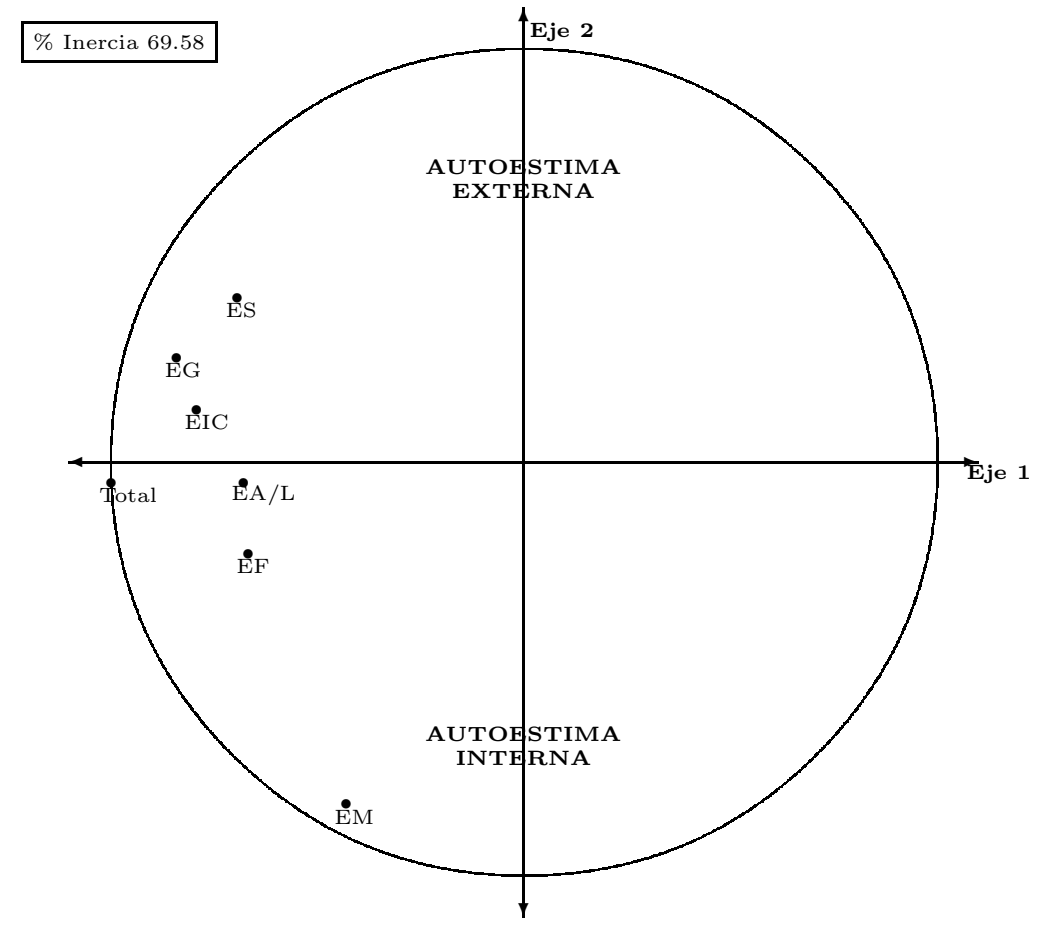

Figura 7: Círculo de correlaciones del Análisis de Componentes Principales para las diferentes subescalas de autoestima.

\section{Modelo 5: individualismo y colectivismo en una Univer- sidad Liberal}

\section{Método}

\section{Participantes}

La muestra de participantes estuvo conformada por 111 alumnos del departamento de Psicología, 79 de la Facultad de Economía y 57 personas trabajadoras en empresas Privadas. La representatividad por sexo se observóde la siguiente forma, 74 de sexo Masculino y 163 del Femenino.

\section{Instrumento}

El instrumento utilizado fue el INDICOL desarrollado por Triandis (1995).

\section{Modelos de análisis}

Análisis de Componentes Principales (ACP). 


\section{Procedimiento}

Los cuestionarios fueron administrados en los horarios y lugares de clase correspondientes de los alumnos, y en los lugares de trabajo, para la muestra laboral. Las variables que se obtienen a partir de la prueba de Triandis permite observar al individualismo y al colectivismo tanto en forma de actitudes como de conductas, por lo que las variables tomadas en el área de actitudes fueron Individualismo Vertical (IV), Individualismo Horizontal (IH), Colectivismo Vertical (CV) y Colectivismo Horizontal $(\mathrm{CH})$; y en el área de conductas estas mismas variables se identifican con una $\mathrm{T}$ al frente, es decir, TIV es individualismo vertical conductual, TIH corresponde a individualismo horizontal conductual, TCV a colectivismo vertical conductual, y TCH a colectivismo horizontal conductual.

\section{Resultados y discusión}

Como se puede observar en la Figura 8, el modelo que se genera de las variables de individualismo y colectivismo tanto el área conductual como la de actitudes de cada variable se corresponden en sus constructos hipotéticos, con lo cual se observa al Individualismo Horizontal tanto conductual como actitudinal en el primer eje, luego al Colectivismo Horizontal en el segundo eje, en el tercero al Colectivismo Vertical y finalmente al Individualismo Vertical. Asimismo, se aprecia que el modelo planteado por Triandis (1995) se comprueba en la realidad al ser evaluado por medio de una investigación de campo. Según Triandis la combinación de las variables verticales y horizontales sobre las de individualismo y colectivismo dan como resultado la comprensión de las personalidades alocentricas (colectivistas en culturas individualistas) e ideocéntricas (individualistas en culturas colectivistas).

Como se advierte en la Figura 8, la Componente 1 está determinada por lo que se denomina Interdependencia - Independencia, mientras que la Componente 2 se comprende bajo los conceptos de Sí Mismo Igual - Sí Mismo Desigual (Chen et al., 1997).

\section{Discusión general}

La aproximación multivariable formalizada en cada uno de los modelos emergente, permitió una óptica hacia útil que resulta representar a los individuos en un espacio de pocas dimensiones perdiendo el mínimo de información (Castillo et al.; 1997)

Cada modelo resalta características importantes y particulares en la aplicación del enfoque multivaraido, pero todos permiten comprender la necesidad de contar con una rápida referencia visual de las variables, que permita reunir el máximo de datos en pocas componentes sobre un plano o círculo, facilitando las decisiones metodológicas a seguir en cada uno de los casos, en el análisis inferencial de las variables.

Como se pudo apreciar a través de la presentación y exploración de cada uno de los cinco modelos, éstos encuentran una vía de validez y fiabilidad sin importar el área sobre la que se fundamentan sus constructos, con lo que se cumple el objetivo obtener, gracias a la ayuda de herramientas estadísticas, la verificación contra la realidad de los modelos que los diferentes teóricos plantean como fruto de las observaciones, experiencias y estudios que han marcado su vida profesional. 


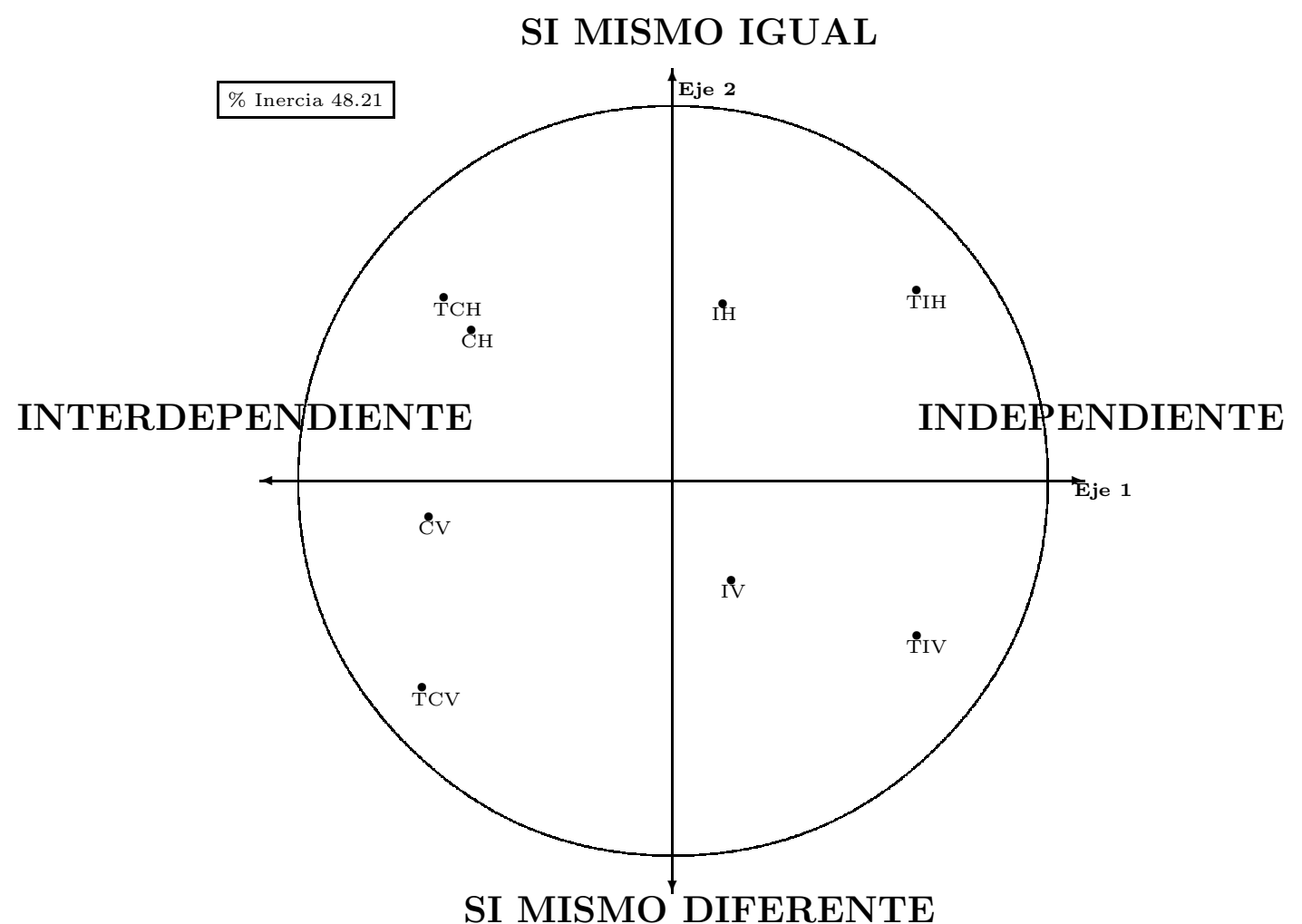

Figura 8: Modelo de Individualismo y Colectivismo derivado del círculo de correlaciones del Análisis de Componentes Principales.

Los resultados llevan a considerar de gran utilidad las técnicas propias del análisis multivariado de datos, tanto para la generación, como la comprobación de los modelos, pues estos pasan a consolidar las teorías que fungen como pilares de las complejas variables que rodean a las ciencias preparadigmáticas de la conducta, como la psicología en sus diversos campos experimentales y aplicados.

De esta forma y siguiendo los consejos de Kurt Lewin, estas ciencias preparadigmáticas avanzaran a medida que las teorías que presentan puedan explicar la conducta en sus diversas manifestaciones, con modelos más simples, los cuales a su vez serán significativamente claros y distintos en su exploración de las variables que conforma al organismo y su ambiente, llegando a consolidar la famosa máxima de este psicólogo social guestaltista, "no hay nada más práctico que una buena teoría"; y no hay mejor teoría que aquella en que los datos hablan por sí misma. 


\section{Referencias}

Alder, H. (1995) Pensar para la Excelencia. EDAF, Madrid.

Anastasi, A.; Urbina, S. (1998) Tests Psicológicos. Prentice Hall, México.

Baron, R. (1996) "The Baron emotional quotient inventory (EQ-i): a test of emotional intelligence", Multi-Health Systems, Toronto.

Castillo, W.; González, J.; Rodríguez, O.; Trejos, J. (1997) Fundamentos de Análisis Multivariado de Datos. Universidad de Costa Rica, San José: .

Chen, C.; Meindl, J. R.; Hunt, R. O. (1997) "Testing the effects of vertical and horizontal collectivism: A study of reward allocations preferences in China", Journal of Cross Cultural Psychology 28: 44-70.

Instituto de Investigaciones Educativas (1970) Escalas programa universitario de pruebas de evaluación. Universidad del Valle de Guatemala, Guatemala.

Kolb, D. A.; Rubin, I. M.; McIntyre, J. M. (1977) Psicología de las organizaciones: Problemas contemporáneos. Prentice-Hall, México.

Leahey, T. (1982) Historia de la psicología. Debate, Madrid.

Luthans, F.; Hodgetts, R. M.; Rosenkrantz, S. A. (1988) Real Managers. Balliger, Cambridge MA.

Otis, A. S. (1987) Tests Otis Autoaplicados de Habilidad Mental. Universidad del Valle de Guatemala, Guatemala.

Pope, A. W.; McHale, S. M.; Craighead, W. E. (1988) Self-esteem enhancement with children and adolescents. Pergamon, Nueva York.

Popper, K. R. (1934) The logic of scientific discovery. Hutchinson, Londres.

Rotter, J. B. (1966) "Generalized expectancies for internal versus external control reinforcement", Psychological Monographs, 80 (609).

Seijas, C. R. (1998a) Del Azar al Hacer en busca de la Excelencia Académica: Predicción del desempeño de los estudiantes de psicología de la Universidad Francisco Marroquín. Tesis del Departamento de Psicología, Universidad Francisco Marroquín, Guatemala.

Seijas, C. R. (1998b) "Estilos de aprendizaje y lateralidad cerebral: validez de constructo, empírica y de diagnóstico, sobre la base de la orientación vocacional". (Documento Interno) Universidad Francisco Marroquín, Departamento de Psicología, Guatemala.

Seijas, C. R. (1999a) "Autoestima en los estudiantes de la facultad de economía". (Documento Interno). Universidad Francisco Marroquín, Departamento de Psicología, Guatemala.

Seijas, C. R. (1999b) "Del azar al hacer II: El perfil de los estudiantes de psicología". (Documento Interno). Universidad Francisco Marroquín, Departamento de Psicología, Guatemala.

Seijas, C. R. (1999c) "Individualismo y Colectivismo en una Universidad Liberal". (Documento Interno). Universidad Francisco Marroquín, Departamento de Psicología, Guatemala.

Salomón de Dougerthy, H. (1989) Predicción del rendimiento académico del estudiante de primer ingreso a la universidad. Tesis inédita de Licenciatura Educación, Universidad del Valle de Guatemala, Guatemala.

Triandis, H. C (1995) Individualism and collectivism. Westview, Boulder CL.

Weinstein, C.E.; Palmer, D.R.; Schulte, A.C. (1987) LASSI: Learning and Study Strategies Inventory. R.H. Publishing Co., Clearwater, Fl. 